\title{
Prácticas Docentes para una Pedagogía Inclusiva en Educación Primaria: Escuchando las voces del Profesorado
}

\author{
Inmaculada Orozco y Anabel Moriña \\ Universidad de Sevilla - España
}

\section{RESUMEN}

Este artículo tiene como propósito explorar las prácticas de veinticinco docentes de Educación Primaria que desarrollan una pedagogía inclusiva. En concreto, tratamos de responder a tres preguntas de investigación: 1) ¿Cuáles son los elementos didáctico-organizativos y las estrategias metodológicas y afectivas que pone en práctica un docente inclusivo? 2) ¿Cuáles son los roles del profesorado y del alumnado? 3) ¿Cuáles son los sistemas de evaluación y autoevaluación que emplea? La metodología ha sido cualitativa, con un diseño multicaso. La información ha sido recogida mediante una entrevista semi-estructurada y analizada con un sistema de categorías y códigos inductivo. Los resultados están organizados en tres temas: estrategias metodológicas y afectivas y otros elementos didácticosorganizativos; roles del profesorado y de los estudiantes; evaluación y autoevaluación. Entre los principales hallazgos cabe resaltar que los participantes emplean estrategias metodológicas variadas, activas y afectivas, el docente desempeña el rol de acompañante y el alumnado de protagonista, y realizan evaluación y autoevaluación auténticas. Las conclusiones dejan ver que estas acciones son oportunidades para continuar investigando sobre otras que den sentido a los procesos educativos.

Palabras Clave: Pedagogía inclusiva, prácticas docentes, profesorado, Educación Primaria, investigación cualitativa.

\section{Teaching Practices for an Inclusive Pedagogy in Elementary Education: Listening the Teachers' voices}

\section{ABSTRACT}

The purpose of this article is to explore the practices of twenty-five primary school teachers who develop inclusive pedagogy. Specifically, we try to answer three research questions: 1) What are the didactic-organizational elements and the methodological and affective strategies that an inclusive teacher puts into practice? 2) What are the roles of teachers and students? 3) What are the evaluation and selfevaluation systems used? The methodology has been qualitative, with a multicaso design. The information has been collected through a semi-structured interview and analyzed with a system of categories and inductive codes. The results are organised into three themes: methodological and affective strategies and other didactic-organisational elements; roles of teachers and students; evaluation and selfevaluation. Among the main findings it is worth highlighting that the participants use varied, active and affective methodological strategies, the teacher plays the role of accompanist and the student body of protagonistand carry out authentic evaluation and selfevaluation. The conclusions show that these actions are opportunities to continue research on others that give meaning to educational processes.

Keywords: Inclusive pedagogy, practices, teachers, Primary Education, qualitative research.

\section{Introducción}

El modelo de educación inclusiva supone convivir con las diferencias y alude a los procesos políticos, sociales y culturales que ocurren en las escuelas y los sistemas educativos para lograr la presencia, el aprendizaje, la participación y el éxito de todos (Ainscow y Messiou, 2018).

Desde este modelo se propone que las escuelas sean espacios que respondan a la diversidad y en el que se reconozcan que todo niño puede aprender, ya que sus capacidades dependen de las oportunidades que se pongan a su disposición (Susinos y Pa- rrilla, 2013; Tonnuci, 2017). La educación inclusiva requiere una mirada más profunda y sensible sobre su significado porque no es una necesidad o un sueño, sino un derecho que ninguna institución debe cuestionar o ignorar (Haug, 2017).

No obstante, es habitual encontrar centros educativos donde una minoría continúa en los márgenes de la exclusión porque recibe una enseñanza diferente (Echeita, 2017; Florian y Beaton, 2018). De hecho, en las escuelas españolas algunos apoyos continúan realizándose fuera del aula ordinaria y no existe una cultura institucional sólida que permita introducir transformaciones metodológicas profundas (Sandoval, Simón y Echeita, 2018). En 
este entramado, deben existir reformas por parte del profesorado para que sea un apasionado en su profesión, erija procesos de reflexión-investigación-acción, luche por la dignidad de cada niño (Arnaiz, De Haro y Guirao, 2015) y valore la diversidad como una oportunidad para buscar nuevos enfoques, en lugar de identificar problemas en "algunos" estudiantes (Sagner-Tapia, 2018; Tonnuci, 2017). Por estas razones, se necesita una pedagogía de la diversidad que repiense los procesos educativos, las personas y los colectivos que son sistemáticamente olvidados (Calderón, Calderón y Rascón, 2016). De este quehacer se encarga la pedagogía inclusiva.

\section{La pedagogía inclusiva y la práctica docente}

La pedagogía inclusiva se centra en analizar qué hace un buen docente, cómo lo hace y por qué opta por la participación de todos en la vida del aula ordinaria, sin excepción, en lugar de focalizar la praxis en la mayoría (Florian, 2014). Esta filosofía según Rouse (2009) va a ser posible y depender de aquello que el profesorado "crea" (expectativas y confianza en los alumnos), "sepa" (sobre cuestiones teóricas, políticas y legislativas) y "haga" (traducir los conocimientos en acciones). En este artículo nos vamos a centrar en las acciones. Spratt y Florian (2015) definen las acciones o prácticas como aquellas posibilidades que un docente origina para que todos los niños aprendan, participen, construyan el conocimiento, progresen y se sientan en igualdad de condiciones, sin excepción. Estas actuaciones se caracterizan por emplear una variedad de estrategias metodológicas en el aula ordinaria centradas en el alumnado que benefician a todos los estudiantes y los implica de forma activa porque se ajustan a sus necesidades, plantean retos, estimulan la creación de entornos de interacción y colaboración, apoyan la motivación, el compromiso, el acceso a la información y el alcance de los objetivos (Majoko, 2019; Woodcock y Hardy, 2017).

Desde la pedagogía inclusiva se desafía a que cualquier docente aprenda de las diferencias y sea sensible a la diversidad, las formas de organizar el aprendizaje y acoger los puntos de vista de los estudiantes (Messiou et al., 2016). Sin embargo, la investigación educativa y la legislación no responden a las barreras que existen en los centros educativos y las prácticas docentes reales (Echeita, 2017), dando lugar a que existan múltiples revisiones teóricas sobre la inclusión, pero pocas investigaciones pragmáticas para saber cómo el profesorado puede promulgar la pedagogía inclusiva con certeza en un aula a partir de las buenas prácticas de otros (Souto-Manning, 2017). Estas son las razones principales que nos impulsan a explorar las acciones del profesorado de Educación Primaria.

\section{Revisión de prácticas efectivas desde la pedagogía inclusiva en Educación Primaria}

Hay estudios donde se ha desvelado que las actividades que generaron mayor inclusión en Educación Primaria son aquellas que están basadas en el Diseño Universal de Aprendizaje (Meyer, Rose y Gordon, 2014), la Teoría de las Inteligencias Múltiples y los estilos de aprendizaje (Elder, Damiani y Oswago, 2016), el juego (Jay y Knaus, 2018), el Aprendizaje-Servicio (Billig, 2017), el Aprendizaje Basado en Problemas (ABP), las simulaciones, Flipped Classroom (Loizou-Raouna y Lee, 2018), el aprendizaje dialógico (Chan y Lo, 2017), el aprendizaje cooperativo (Moliner, Moliner y Sanahuja, 2018), los proyectos de trabajo o en el uso de diferentes recursos tecnológicos (Gómez y Badia, 2016). Otras investigaciones muestran la creación de un ambiente seguro, dando feedback y basándose en las experiencias (Westbook y Croft, 2015), durante los recreos, a través del ejercicio físico, flexibilizando el tiempo (Dyson, 2014), con la co-enseñanza, el apoyo entre iguales y los agrupamientos heterogéneos (Elder et al., 2016).

Por otro lado, hay evidencias acerca del componente afectivo en el aprendizaje. Es decir, con el vínculo emocional que el docente establece con los estudiantes, así como con su propia motivación e identidad (Brien, Blue y Rowlands, 2017). Este confía en la capacidad de aprender y se preocupa por cómo y qué aprenden los estudiantes (Chan y Lo, 2017) desde una perspectiva constructivista, apoyando a todos a partir del aprendizaje por descubrimiento (Klibthong y Agbenyega, 2018) e involucrándose con ellos como uno más (Nind y Lewthwaite, 2018). Además, el docente se detiene a conocer a los estudiantes, fomenta interacciones positivas con y entre ellos (Yin, Huang y Chi, 2017) y es responsable de encontrar maneras de asegurar que exista un fuerte compromiso social y todos los niños sean felices, así como una variedad de experiencias ricas dentro y fuera del contexto escolar (Sheehy y Budiyanto, 2015). De esta manera, se posiciona como agente de cambio que puede transformar las escuelas al reflexionar individual y colectivamente sobre lo que hace (Juma, Lehtomäki y Naukkarinen, 2017; Bjørnsrud y Nilsen, 2019).

En el ámbito de la evaluación, las investigaciones de Florian y Beaton (2018) o Meyer et al. (2014), manifiestan que el docente inclusivo lleva a cabo una evaluación formativa y de carácter continuo que favorece la metacognición, permite aprender del error, estar más motivado y ser flexible. Se trata de implementar una evaluación auténtica que atienda a la autoevaluación y fortalezca tanto a él como a los niños (Bourke, 2016). Para ello, el maestro actúa como un observador participante y origina andamiajes, graba vídeos de sus clases, realiza diarios personales, registra conversaciones dialógicas, escucha sus voces, cambia sus planes y les hace responsables y partícipes en el proceso y la toma de decisiones (Petrie, Devcich y Fitzgerald, 2018).

Este concibe que el resultado del aprendizaje de todos los estudiantes es una responsabilidad compartida, así que da la bienvenida a las opiniones de otros, se preocupa por obtener la mayor información posible de sus discentes, amplía su conocimiento, unifica criterios, planifica y evalúa la docencia en compañía de docentes especializados (Gómez-Zepeda, Petrenas, Sabando y Puigdellívol, 2017) y estableciendo un fuerte compromiso entre la escuela y la familia (Vigo y Soriano, 2015).

En definitiva, con este trabajo pretendemos llenar un vacío existente en la literatura a nivel práctico sobre cómo un docente puede actuar en las escuelas para hacerlas más humanas, dialógicas y accesibles en la etapa de Educación Primaria. En concreto, tenemos como propósito descubrir y mostrar cuáles son las estrategias de enseñanza, afectivas y emocionales de estos docentes y cómo son capaces de adaptarse y responder a las demandas de una escuela inclusiva.

A diferencia de la mayoría de las investigaciones (Florian y Beaton, 2018; Juma et al., 2017; Yin, et al., 2017), no estudiamos de manera parcelada los factores que pueden favorecer la inclusión, como es el caso de las estrategias didácticas y afectivas o la evaluación entre otras, sino que analizamos detalladamente cada una de ellas desde una visión holística y centrada en el docente. El objetivo general que nos planteamos es analizar las prácticas del profesorado que lleva a cabo una pedagogía inclusiva. Para ello nos preguntamos: 1) ¿cuáles son los elementos didáctico-organizativos y las estrategias metodológicas y afectivas que pone en práctica un docente inclusivo?, 2) ¿cuáles son los roles del profesorado y del alumnado?, 3) ¿cuáles son los sistemas de evaluación y autoevaluación que emplea? 


\section{Método}

Este estudio forma parte de una investigación más amplia en el que pretendíamos estudiar qué hace, cómo y por qué el profesorado de Educación Primaria que desarrolla una pedagogía inclusiva ${ }^{1}$. Se utilizó una metodología de investigación cualitativa. En concreto, se empleó la metodología de estudios multicasos (Stake, 2006).

\section{Participantes}

La selección de participantes fue el resultado de un muestreo intencional por conveniencia y criterial. En concreto, para garantizar la idoneidad de los participantes formulamos cinco criterios para su selección:: 1) docentes de Educación Primaria que desarrollen una pedagogía inclusiva, 2) diversidad de edad, 3) diversidad de género, 4) diversidad de años de experiencia docente, y 5) disponibilidad en la participación y tiempo.

Acudimos a dos centros de formación del profesorado (CEP). Los asesores de estos centros fueron los que nos facilitaron contactos de profesores que estaban desarrollando una pedagogía inclusiva en Educación Primaria. Además, recurrimos al muestreo no probabilístico de bola de nieve (Cohen, Manion y Morrison, 2000), contactando con aquellos "colegas" recomendados por los participantes iniciales. La muestra al final quedó compuesta por 25 docentes de once escuelas públicas de esta etapa. $\mathrm{Su}$ perfil se caracterizaba por haber impartido docencia en escuelas donde existía un alto índice de alumnado en situación de vulnerabilidad y riesgo de exclusión social. Había una prevalencia en género femenino: 18 mujeres (72\%) y 7 hombres (28\%). La media de edad del profesorado fue de 43.96 años y variada: cinco profesionales de 34 a 39 años (20\%), nueve de 40 a 49 años (36\%) y ocho de 50 a 57 años (32\%). Respecto a la experiencia docente, de los veinticinco, dos habían ejercido entre 5 y 10 (8\%) años, seis entre 10 y 15 (24\%), siete entre 15 y 20 (28\%), dos entre 20 y $25(8 \%)$, cuatro entre 25 y 30 (16\%), tres entre 30 y 35 (12\%) y solo uno había desempeñado su profesión durante más de 35 años (4\%).

\section{Instrumento y procedimiento}

Elaboramos un guion de entrevista semi-estructurada con preguntas dedicadas a estudiar cuáles eran las prácticas docentes. Estas fueron algunas cuestiones que se abordaron de manera flexible en los encuentros: ¿qué metodología emplea en su aula para originar un buen clima e incluir a todos los estudiantes?, ¿cómo motiva a todos los alumnos para que disfruten y se impliquen mientras aprenden?, ¿qué recursos utiliza?, ¿cómo organiza el ambiente de aprendizaje para que ellos accedan al conocimiento y participen activamente?, ¿cuál es su rol en el aula?, ¿y el de sus alumnos?, o ¿cómo evalúa el aprendizaje de todo su alumnado? Para garantizar la idoneidad del guion, este fue pilotado con profesores que no participaron en el estudio.

Las entrevistas fueron individuales y cara a cara. Tuvieron una duración promedio de 75 minutos. Toda la información fue grabada en horarios no lectivos para el profesorado. Estas una vez transcritas fueron devueltas a sus participantes correspondientes por correo electrónico para que validaran las mismas, realizaran los cambios que consideraban oportunos y garantizaran que estaban conformes con los datos.

\section{Análisis de datos}

Creamos un sistema de categorías y códigos inductivo que facilitó la comprensión de los diferentes datos recogidos y dar sentido a la información (Miles y Huberman, 1994). Este fue construyéndose a partir de la lectura de las transcripciones de las entrevistas, las cuales fueron analizadas a mano, y posteriormente incorporadas en el programa MAXQDA 12. Este programa nos permitió manejar el gran volumen de información con la que contábamos. Para garantizar la rigurosidad del análisis toda la información fue analizada simultáneamente por las dos autoras del artículo.

En el estudio emergieron cuatro categorías: creencias, conocimientos, diseños y acciones. Para este artículo la categoría incluida fue la referida a acciones. Los códigos utilizados en este análisis fueron trece: metodología, adaptaciones, organización del ambiente, agrupamientos, motivación, recursos, estrategias, estrategias fundamentales, habilidades inclusivas, rol docente y papel del alumnado, evaluación, descubrimiento del buen trabajo y autoevaluación docente. Los subcódigos que emergieron de dicho análisis fueron dieciséis: variedad metodologías, tutorización entre iguales, coordinación con otros profesionales, adaptaciones intrínsecas, organización y agrupamientos diversos, recursos (comunitarios, humanos y tecnológicos), múltiples roles, alumnado en el centro del aprendizaje, vínculo afectivo, numerosos instrumentos, herramientas tecnológicas y familias, exámenes, reflexión diaria, confianza, felicidad y formación.

\section{Cuestiones éticas de la investigación}

Para proteger los derechos de los participantes utilizamos un consentimiento informado donde se comunicaba que su colaboración en el estudio era voluntaria y estaba amparada de posibles riesgos o daños, siempre respetando y garantizando su privacidad y confidencialidad. Además, para respetar su anonimato se emplearon abreviaturas y números que no revelaban su identidad real.

\section{Resultados}

En este apartado se muestra una información parcial acerca de las cuatro dimensiones que componen un estudio más amplio centrado en las creencias, los conocimientos, los diseños y las acciones. En concreto, en esta ocasión se presentan cuáles fueron las prácticas del profesorado de Educación Primaria que desarrolla una pedagogía inclusiva, dando respuesta la última dimensión, es decir, a qué, para qué, con quién y cómo la hacen posible. En este estudio surgieron tres temas: las estrategias metodológicas y afectivas y otros elementos didácticos-organizativos; roles del profesorado y los estudiantes; y evaluación y autoevaluación.

\section{Las Estrategias Metodológicas y afectivas y otros Elementos Didáctico-Organizativos: Hacia una Práctica con Todos y Para Todos}

\section{a) Metodologías y estrategias metodológicas}

Las metodologías que utilizaban todos los docentes que participaron en este estudio fueron participativas, globales, interactivas, constructivistas y activas. Entre ellas destacaban el aprendizaje cooperativo, la gamificación, el ABN, los grupos

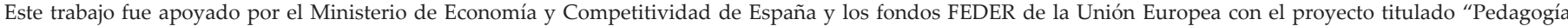
Inclusiva en la Universidad: Narrativas del Profesorado" [EDU2016-76587-R]. 
interactivos, el juego dramático, la Inteligencia Emocional, la Neurociencia y la convivencia pacífica. También ofrecían distintas rutas para acceder a la información que daban respuesta a la diversidad: la ayuda de un compañero, llevarse la información a casa o navegar por Internet. En general, no recurrían a una única metodología, sino que eran varias.

ENT.9: Un poquito de todo porque una metodología le va a venir bien a unos y a otros no, entonces tienes que ir cambiando.

A través de estas metodologías explicaban que todas las voces eran cuestionadas, escuchadas y válidas en el gran grupo. Se posicionaban como compañeros de viajes y más que dar respuestas, generaban preguntas.

ENT.21: Trabajo mucho los procedimientos y corregirnos en grupo, sobre todo, que todo lo que pase en la clase y en el trabajo que hagamos tenga una reflexión y un pensamiento.

El profesorado también potenciaba la competencia de "aprender a aprender", daba libertad para que diseñaran sus propias actividades, se mostraba como modelo, hacía uso del método científico y las nuevas tecnologías, pero, sobre todo, cuidaba las habilidades emocionales de todos los niños.

ENT.24: En tutoría trabajamos las emociones con un cuaderno de pensar, una estrategia y un recurso que facilita mucho el trabajo a nivel de grupo-clase. Ellos van apuntando las incidencias que han tenido, cómo se han sentido y qué les ha pasado para debatirlo y solucionarlo.

Otras de las estrategias que destacaron eran decidir las normas entre todos, emplear la técnica simple de aprendizaje cooperativo denominada "el saco de dudas" y utilizar la maleta viajera. La estrategia más nombrada fue la tutoría entre iguales. Los profesionales sentían que al usarla su trabajo era más efectivo, y a la vez, tejían redes de confianza, fomentaban la ayuda, la empatía, la libertad de hablar, la convivencia y el éxito compartido.

El juego y el Mindfulness fueron otros de sus aliados para captar el interés del alumnado y hacer que este aprendiera disfrutando. Para iniciar el proceso de aprendizaje, sabían que tanto la mente como el cuerpo tenían que estar predispuestos.

ENT.19: Cada 20 o 25 minutos parar y hacer juegos de equilibrio o ritmo, para desconectar un poquito porque según la Neurociencia después de 20 minutos empezamos a flaquear. Tú lo único que haces es parar, pero vamos no pierdes, inviertes y ganas en concentración y atención.

Por otro lado, el trabajo compartido del profesorado de Pedagogía Terapéutica y otros especialistas del centro junto al tutor de aula fue otra de las estrategias a la que recurrían. Consideraban que se trataba de una pareja pedagógica porque ambos profesionales se mostraban como referentes y juntos reflexionaban sobre su praxis. El especialista no se encargaba de hacer una tarea paralela con algún niño concreto, sino que en el mismo espacio actuaba como un docente más.

ENT.12: El maestro de apoyo entra en el aula trabaja con el equipo y no sólo con esta persona.

De este modo, las adaptaciones curriculares eran no significativas y se llevaban a cabo porque la ley exigía esta tarea, aunque estas adaptaciones ya iban intrínsecas en las distintas me- todologías porque permitían dar respuesta a la diversidad sin hacer una dinámica distinta con una minoría.

ENT.8: Las adaptaciones no significativas las utilizo a diario, porque eres tú el que debes adaptarte a cada niño, a su manera de aprender y mediar en cómo aprende cada niño.

\section{b) El componente afectivo en el aprendizaje}

Los participantes en este estudio sabían que sin motivación no hay aprendizaje. Para ellos no tenía sentido intentar que el alumnado asimilara una serie de contenidos si no le encontraban vinculación con su vida. Con el fin de lograrlo creían en lo que hacían, incorporaban los contenidos de menor a mayor nivel de dificultad, usaban el Aprendizaje-Servicio y la relajación, jugaban con sus estudiantes, les asignaban responsabilidades, tenían altas expectativas y confiaban en sus estudiantes, reconocían sus propios errores, reflexionaban en grupo, tenían una postura calmada y positiva, y se detenían seriamente a abordar las emociones para que sus estudiantes fueran resilientes a lo largo de su vida. Los docentes consideraban que las relaciones afectivas favorecen el aprendizaje y debían cuidarse porque era un ingrediente básico para que las aulas fueran realmente humanas.

En esta línea, sobresalieron otras estrategias como la escucha para construir juntos y dejar a los estudiantes que eligieran qué contenidos querían aprender. De esta forma, todos se sentían parte del proyecto de aula porque se consideraban un grupo y se sentían importantes. No obstante, lo más relevante que destacaron fue saber mirar a cada niño como merece, como una persona llena de posibilidades.

ENT.21: Haciendo que ellos sientan que tú lo has visto; que ellos se sientan vistos y mirados. Las estrategias tienen que ver con eso, con buscarle su sitio y no tanto con las dinámicas.

\section{c) Organización del ambiente de aprendizaje}

El ambiente de aprendizaje concebía la asamblea como el corazón del aula y este no tenía una estructura fija, sino que podía ser cualquier espacio (los pasillos del centro, el patio del recreo o un parque a las afueras) y dependía de las necesidades que fueran surgiendo en el día. Algunas zonas de la clase cuidaban la ayuda entre iguales y los afectos del grupo.

ENT.1: Tengo una pizarra de los sabios, porque si alguna persona no entiende algo, tú tienes derecho mientras estamos en la clase a levantarte con ella y explicárselo para que lo comprenda.

En general, la organización era en gran o pequeño grupo, por parejas e incluso individual, pero intentaban que el mayor tiempo posible se dedicara a los equipos y el gran grupo para que hubiera más puntos de vista y surgieran conversaciones culturales que tejieran los centros de interés y proyectos de trabajo. Los agrupamientos eran flexibles, mixtos y heterogéneos, normalmente compuestos por cuatro o cinco miembros, aunque estaban basados en la complejidad y las diferentes circunstancias del día.

ENT.22: Nosotros somos muy de dejar las sillas apiladas como un bar y en función de lo que vaya demandando se hace uso.

\section{d) Recursos}

Solían ofrecer con mayor frecuencia aquellos que permitían traer la realidad al aula y pertenecían a la comunidad (volun- 
tariado de la facultad, expertos...). De igual modo, hacían uso de otros recursos humanos como los especialistas (Pedagogía Terapéutica, Audición y Lenguaje y Cupo de Apoyo y Refuerzo), los propios niños, las familias y otros docentes. También empleaban recursos del propio entorno como el huerto escolar o la biblioteca, tecnológicos como plataformas digitales y blogs de clase, el ordenador y la pizarra digital interactiva (PDI), así como materiales manipulativos, musicales y audiovisuales (vídeos y fotografías).

ENT.21: Yo tiro de todo el mundo, de todas las personas que tanto yo como el alumnado conocemos y saben de lo que estamos trabajando para que nos asesore y forme.

\section{Los Roles de los Estudiantes y el Profesorado en la vida del Aula: Nuevas Funciones y Relaciones para la Participación de Todos}

El profesorado afirmaba que no tenía un único rol, sino que eran múltiples los papeles que desarrollaba a lo largo de su práctica. La gran mayoría se definieron como facilitadores y responsables de empoderar y sacar el máximo potencial de cada niño porque generaban situaciones donde se convivía con la diversidad y todo el alumnado iba descubriendo que podía elegir cómodamente el itinerario que quería seguir en su aprendizaje.

ENT.8: Yo dejo que cada personalidad aflore. Si un niño soluciona un conflicto con una carta de arrepentimiento, otro lo puede solucionar de otra manera, por ejemplo, dándole un beso o una caricia.

Por otro lado, en general, los profesionales cuidaban sus gestos, se mostraban accesibles y eran cercanos, transparentes, afectivos y dedicaban su tiempo a escuchar a los estudiantes, asumiendo el papel de mediadores y, sobre todo, de acompañantes. Esta última postura fue una de las más nombradas porque permitía a los niños sentirse seguros al saber en cualquier momento podían acudir a un referente adulto.

ENT. 12: Yo dejo que los niños vayan solos. Yo estoy un poco por detrás, por si necesitan ayuda, surge algún problema..., pero, sobre todo, les dejo que se equivoquen.

Por tanto, el alumnado era el protagonista en el acto educativo y desempeñaba un rol participativo, reivindicativo y reflexivo, y tenía el derecho a deshacer, aprender y desaprender junto al docente. Así, eran dueños de su proceso, se implicaban y comprometían diariamente para que en el aula ocurriese aquello que realmente les pertenecía e interesaba hacer.

De esta manera, los niños estaban en continuo movimiento y tendían a construir el aprendizaje poniendo en duda aquello que el docente planteaba en la clase al intercambiar incertidumbres y opiniones con la ayuda de sus compañeros. Así, conseguía ciudadanos críticos, autónomos, respetuosos, curiosos, creativos y capaces de resistir ante cualquier conflicto que pudiera aparecer en sus vidas.

ENT.1: Ellos están todo el día ocupados haciendo cosas; buscando, informándose, pensando, trabajando, creando, haciendo y moviéndose. No quiero clases calladas, no quiero clases que no participen, no quiero niños que no piensen, no quiero niños que me digan a todo que sí. Quiero debate, quiero creación.

En definitiva, los docentes se preocupaban por establecer un vínculo sano y humano con su alumnado, dedicaban tiempo para obtener información y conocerlos en profundidad por ellos mismos desde diferentes vías. Esto lo hacían a través de entrevistas y cuestionarios a la familia, observándoles en silencio en distintos momentos (especialmente en los recreos), estableciendo encuentros con otros profesionales, realizando sociogramas y tutorías con el grupo, y creando dinámicas cooperativas, afectivas y de cohesión grupal como las cajas de vida.

ENT.7: Si yo no conozco a mis niños no tiene sentido nada de lo que hago. Me refiero, yo llego a clase el primer día y no puedo explicar sin saber qué le gusta a cada uno, de qué carece, o si tiene problemas familiares o no.

\section{Evaluación y Autoevaluación: Caminos Entrecruzados que Per-} miten la Mejora Docente

Para el profesorado evaluar significaba reflexionar y mejorar continuamente sobre lo que se hace en la escuela a partir de las voces de los estudiantes. Los participantes evaluaban a partir del interés, la disposición y el esfuerzo. Ellos emplearon instrumentos variados como la observación sistemática del trabajo diario, las rúbricas, el diálogo, las fotografías y los vídeos, los cuadernos de campo, las exposiciones orales, los trabajos en grupo y el portafolio. Además, recurrían a herramientas tecnológicas como Idoceo y otras gamificadas como Plickers, Classcraft, Kahoot y Classdojo que le permitían coevaluar, atender a la diversidad, centrarse en la vida cotidiana y evaluar por competencias.

Algunos que realizaban exámenes, pedían a su alumnado que el mismo lo corrigiese en clase con otros para que fuese tomando conciencia de cuáles eran sus debilidades. Además, valoraban profundamente, por ejemplo, si un niño había sido capaz de ayudar a otro compañero o cómo había resuelto distintos retos personales que se le iban presentando.

ENT.25: Intento registrar formas personales de abordar el aprendizaje, no sólo si consigue o no consigue determinado objetivo, sino cómo lo hace y en qué tiene dificultades para transformarlas en fortalezas.

Su labor la autoevaluaban de la misma forma que evaluaban el progreso de sus estudiantes, añadiendo a su vez la opinión de estos, el diario personal, los anecdotarios, la transcripción y el análisis de las conversaciones, las tutorías con la familia e intercambiando experiencias con docentes de otros centros educativos.

ENT.12: Tengo un diario de lo que voy más o menos haciendo. Después comparto mis experiencias con otros profesores que no son de este centro para analizar lo que hacemos y de ahí sacar conclusiones para ir probando cosas.

Cuando se les pedía que explicaran cómo descubrían que habían hecho un buen trabajo con cada niño respondieron que lo hacían siendo exigentes con ellos mismos, estando dispuestos a realizar ajustes necesarios y apreciando que sus estudiantes podían confiar en ellos. En la mayoría de las ocasiones, reconocieron que la satisfacción la contemplaban a corto plazo cuando estaban felices en el colegio, mientras que a largo plazo sentían que siempre podían haberlo hecho mejor.

ENT.13: Yo me siento muy satisfecha cuando los veo felices. No sé cuántos contenidos habrán aprendido, pero cuando he visto que están bien y felices, sé que lo he hecho bien. 
En este sentido, la mejora le daba al profesorado aliento para transformarse y no permanecer en la zona de confort. Para ello, eran humildes, escuchaban al prójimo, se formaban permanentemente junto a otros profesionales con las mismas inquietudes, realizaban lecturas, visitaban canales y blogs educativos, investigaban continuamente y aprendían de los errores.

\section{Conclusiones y Discusión}

El profesorado de Educación Primaria no debe tomarse las acciones incluidas en este artículo como unas simples recetas a seguir, sino como posibles horizontes sobre los que construir una educación y sociedad más justa que vele por el cumplimiento de los derechos de todo el alumnado. Los participantes de este estudio han dado fe de sus buenas prácticas y estas pueden ser las esperanzas que otros necesiten para construir unos espacios de aprendizaje más accesibles y humanos. Del mismo modo, los profesionales podrán demandar formación y realizarla en esta línea, elegir aquellas propuestas que más se adaptan a la realidad del entorno donde desempeñan su labor y con la que más cómodos se sientan, experimentarlas, debatirlas con sus compañeros y continuar investigando sobre otras formas de hacer pedagogía inclusiva.

Las metodologías y estrategias que emplean son activas, democráticas y variadas. En el estudio de Majoko (2019) se resalta que este tipo de estrategias puede beneficiar a todo el alumnado y el profesorado debería recibir una formación previa y permanente sobre cómo ponerlas en práctica. Nuestros resultados coinciden, además, con otros previos que relevan que el juego (Jay y Knaus, 2018), el aprendizaje por descubrimiento, implicar a la familia (Vigo y Soriano, 2015), el Aprendizaje-Servicio (Billig, 2017), el ABP, el aprendizaje cooperativo, Mindfulness, las conversaciones culturales y el aprendizaje dialógico, los proyectos de trabajo, los grupos interactivos (Flecha y Soler, 2013), la escucha activa, cuidar el cerebro, los afectos y las emociones (Westbook y Croft, 2015), usar las nuevas tecnologías (Gómez y Badia, 2016), la tutoría entre iguales, la co-enseñanza y la presencia de dos figuras adultas (Elder et al., 2016), pueden ser algunas claves para lograr espacios más amigables para todos.

Las actividades diferenciadas para una minoría, según los resultados de nuestro estudio, como son las adaptaciones curriculares no tienen cabida en una escuela inclusiva porque más bien se trata de realizar ajustes razonables que den respuesta a cada niño (Meyer et al., 2014). También, se ha podido apreciar que el espacio es otro educador más y se debe organizar en función de las necesidades del día y la dinámica, con agrupamientos cambiantes (Spratt y Florian, 2015).

Para los participantes, los recursos humanos poseen un valor incalculable para erigir unas acciones comunitarias, pero también consideran que los recursos tecnológicos favorecen el aprendizaje, la participación (Gómez y Badía, 2016) y la comunicación con la familia. Esta suele tomar decisiones en la escuela, es escuchada y forma parte de los proyectos. En este sentido, se ha podido apreciar que las aportaciones sobre las Comunidades de Aprendizaje de Flecha y Soler (2013) corroboran las tertulias dialógicas o los grupos interactivos como buenas prácticas. En nuestro estudio, además de estas, aparecen otras como escuchar y actuar de acuerdo a las propuestas de las familias y el uso de herramientas gamificadas.

Por otra parte, los participantes de nuestra investigación redefinen sus roles, reflexionan y caminan hacia la misma dirección con los especialistas (Gómez-Zepeda et al., 2017). De este modo, las aulas específicas deberían repensarse en las escuelas, poniendo en una balanza los frutos que se pueden extraer si todas las personas conviven en el aula ordinaria. Esto es un paso más en el estudio de Juma et al. (2017) porque los beneficios de la acción colaborativa no solo se pueden comprobar entre un profesor experto y uno principiante como ellos han reflejado, sino entre dos personas que comprenden y reconocen que la educación se basa en el respeto de los derechos humanos y aprender juntos.

Los roles del profesorado se asocian con ser facilitadores de los procesos de enseñanza-aprendizaje y responsables de garantizar una educación personalizada, donde cada estudiante sabe que aporta en la escuela. Además, se preocupan por establecer vínculos afectivos y cálidos con y entre sus estudiantes para conocerlos y crear aprendizajes que tengan sentido para sus vidas. Esto coincide con las conclusiones de Arnaiz et al. (2015), Klibthong y Agbenyega (2018), Yin et al. (2017), quienes evidenciaron que las prácticas efectivas reimaginan el rol docente y cuidan el aspecto humano. En nuestro trabajo, exploramos que un educador inclusivo toma un papel distinto y más que facilitar, acompaña, confía y deja que cada niño descubra y decida qué y cómo quiere aprender. No es suficiente con ofrecer los medios educativos, sino que hay que escuchar, respetar, empatizar y crear condiciones adecuadas desde el compromiso para que se produzca la participación y el progreso de todas las personas.

La evaluación, para ellos, es un proceso que le sirve para repensar su praxis y mejorarla, no para encorsetar o poner en juicio la capacidad del alumnado. En general, hacen uso de un abanico amplio de instrumentos, cuidan el feed-back, las relaciones sociales y no se limitan a un examen (Bourke, 2016). De la misma manera, nuestra investigación coincide con los estudios de Florian y Beaton (2018), Petrie et al. (2018), Budiyanto et al. (2018) y Bjørnsrud y Nilsen (2019) porque el profesorado autoevalúa la práctica a partir de la felicidad de los estudiantes, escuchando sus conversaciones, realizando diarios e intercambiando experiencia con otros profesionales. En este artículo se puede apreciar otras sendas sobre las que transitar para lograr una evaluación y autoevaluación auténtica del aprendizaje como es la formación y utilización de plataformas virtuales.

En síntesis, este trabajo cubre un hueco valioso en la literatura sobre cómo hacer pedagogía inclusiva en la etapa de Educación Primaria y abre un camino sobre nuevas líneas de actuación e investigación. El profesorado debe ser un acompañante afectivo, preocupado por conocer la historia de vida de cada uno y dar respuesta a las distintas formas de ser, pensar y actuar. Con estrategias variadas, activas, reales y centradas en las necesidades e intereses de los estudiantes es posible, pero no hay que olvidar el uso adecuado y continuo de una evaluación y reflexión para mejorar la tarea educativa.

\section{Limitaciones e investigaciones futuras}

En primer lugar, consideramos que la disponibilidad y especificidad de la muestra hizo que la selección de los participantes y el proceso de la recogida de datos se ralentizara porque requería contactar con los centros de profesorado y algunos equipos directivos de las escuelas para que nos aseguraran y facilitaran nombres, así como adaptarse al horario y las circunstancias del profesorado.

Creemos que esta investigación nos ha abierto horizontes para ampliar la mirada en futuras investigaciones y descubrir cómo los profesionales pueden pasar de la teoría a la acción, es decir, hacer pedagogía inclusiva pero también hacia otras etapas educativas, desde Educación Infantil hasta Educación Superior.

En definitiva, sería esencial estudiar en profundidad buenos ejemplos de docentes para llevar a cabo estudios de caso o historias de vida que trazaran la trayectoria que una persona ha experimentado hasta convertirse en un docente inclusivo. Esto 
podría complementarse con grabaciones de vídeo y observaciones en la dinámica de clases y entrevistas a otras voces, como los propios estudiantes.

\section{Referencias bibliográficas}

Arnaiz, P., De Haro, R. y Guirao, J.M. (2015). La evaluación en Educación primaria como punto de partida para el desarrollo de planes de mejora inclusivos en la Región de Murcia, Revista electrónica interuniversitaria de formación del profesorado, 18 (1), 103-122. doi:10.6018/reifop.18.1.214351.

Ainscow, M. y Messiou, K. (2018). Engaging with the views of students to promote inclusion in Education. Journal of Educational Change, 19, 1-17. doi: 10.1007/s10833-017-9312-1.

Billig, S. H. (2017). Implementing Service-Learning in Elementary Schools to Enhance Inclusion. In S. Lavery, D. Chambers y G. Cain (Ed.), Service-Learning, International Perspectives on Inclusive Education. Australia: Emerald Publishing Limited, (pp. 75-94).

Bourke, R. (2016). Liberating the learner through self-assessment. Cambridge Journal of Education, 46(1), 97-111. doi: 10.1080/0305764X.2015.1015963.

Bjørnsrud, H. y Nilsen, S. (2019). Joint reflection on action- a prerequisite for inclusive education? A qualitative study in one local primary/lower secondary school in Norway. International Journal of Inclusive Education, 23(2),158-173. doi:10.1080/13 603116.2018.1427153.

Block, K., Suzanne Cross, S., Riggs, E. y Gibbs, L. (2014). Supporting schools to create an inclusive environment for refugee students, International Journal of Inclusive Education, 18(12), 1337-1355. doi: 10.1080/13603116.2014.899636.

Brien, M., Blue, L. y Rowlands, D. (2017). My best possible learning self: Primary school children's perspectives on happiness and success in the classroom. International Journal of Pedagogies and Learning, 12(1), 1-16. Recuperado de https:// eprints.qut.edu.au/115899.

Budiyanto, Sheehy, K., Kaye, H. y Rofiah, K. (2018). Developing Signalong Indonesia: issues of happiness and pedagogy, training and stigmatisation. International Journal of Inclusive Education, 22(5), 543-559. doi:10.1080/13603116.2017.1390000.

Calderón Almendros, I., Calderón Almendros, J.M. y Rascón Gómez, M. T. (2016). De la identidad del ser a la pedagogía de la diferencia, Teoría de la Educación. Revista Interuniversitaria, 28(1). doi: 10.14201/teoredu20162814560.

Chan, C. y Lo, M. (2017). Exploring inclusive pedagogical practices in Hong Kong primary EFL classrooms, International Journal of Inclusive Education, 21(7), 714-729. doi: 10.1080/13603116.2016.1252798.

Cohen, L., Manion, L. y Morrison, K. (2000). Research methods in education. London, UK: Routledge/Falmer.

Dyson, L. (2014). Teachers' Perspectives and Experiences of the Contexts of Social Inclusion within Elementary School. Journal of the International Association of Special Education, 15(2), 108-117. Recuperado de https://eric.ed.gov/?id=EJ1058320

Echeita Sarrionandia, G. (2017). Educación inclusiva. Sonrisas y lágrimas. Aula abierta, 46, 17-24. doi:10.17811/rifie.46.

Elder, B.C., Damiani, M.L. y Oswago, B.O. (2016). From attitudes to practice: utilising inclusive teaching strategies in Kenyan primary schools, International Journal of Inclusive Education, 20(4), 413-434.doi: 10.1080/13603116.2015.1082648.

Flecha, R. y Soler, M. (2013). Turning difficulties into possibilities: engaging Roma families and students in school through dialogic learning, Cambridge Journal of Education, 43(4), 451465. doi: 10.1080/0305764X.2013.819068.
Florian, L. (2014). What counts as evidence of inclusive education? European Journal of Special Needs Education, 29(3), 286294. doi:10.1080/08856257.2014.933551.

Florian, L. y Beaton, M. (2018). Inclusive pedagogy in action: getting it right for every child. International Journal of Inclusive Education, 22(8), 870-884. doi: 10.1080/13603116.2017.1412513.

Gavish, B. (2017). The Implementation of School Inclusion Practices for Students with Special Needs in Israel: Teachers' Perceptions. International Journal of Disability, Development and Education, 64(5), 544-560. doi:10.1080/1034912X.2017.1310372.

Gómez Domingo, M. y Badia Garganté, A. (2016). Exploring the use of educational technology in primary education: Teachers' perception of mobile techology learning impacts and applications' use in the classroom. Computers in Human Behavior, 56, 21-28. doi: 10.1016/j.chb.2015.11.023.

Gómez-Zepeda, G., Petrenas, C., Sabando, D. y Puigdellívol, I. (2017). The role of the support and attention to diversity teacher (SADT) from a community-based perspective: promoting educational success and educational inclusion for all. Teaching and Teacher Education, 64, 127-138. doi: 10.1016/j. tate.2017.02.002.

Jay, J, A. y Knaus, M. (2018). Embedding Play-Based Learning into Junior Primary (Year 1 and 2) Curriculum in WA. Australian Journal of Teacher Education, 43(1), 112-126. Recuperado de https://ro.ecu.edu.au/ajte/vol43/iss1/7/.

Juma, S., Lehtomäki, E. y Naukkarinen, A. (2017). Scaffolding teachers to foster inclusive pedagogy and presence through collaborative action research, Educational Action Research, 25(5), 720-736. doi: 10.1080/09650792.2016.1266957.

Haug, P. (2017). Understanding inclusive education: ideals and reality. Scandinavian Journal of Disability Research, 19(3), 206217. doi: 10.1080/15017419.2016.1224778.

Klibthong, S. y Agbenyega, J. S. (2018). Exploring Professional Knowing, Being and Becoming through Inclusive Pedagogical Approach in Action (IPAA) Framework. Australian Journal of Teacher Education, 43(3). Recuperado de https://ro.ecu.edu. au/ajte/vol43/iss3/7/

Loizou-Raouna, M. y Lee, K. (2018). A flipped classroom model for inquiry-based learning in cyprus primary education context. In M. Bajié, N. B. Dohn, M. de Laat, P. Jandrié, y T. Ryberg (Eds.), Proceedings of the 11th International Conference on Networked Learning 2018 (pp. 210-217). Lancaster University: Cyprus Ministry of Education and Culture.

Majoko, T. (2019). Teacher Key Competencies for Inclusive Education: Tapping Pragmatic Realities of Zimbabwean Special Needs Education Teachers. SAGE Open. doi:10.1177/2158244018823455.

Messiou, K., Ainscow, M., Echeita, G., Goldrick, S., Hope, M., Paes, I., ... Vitorino, T. (2016). Learning from differences: a strategy for teacher development in respect to student diversity. School Effectiveness and School Improvement, 27(1), 45-61. doi:10.1080/09243453.2014.966726.

Meyer, Rose, D. H. y Gordon, D. (2014). Universal Design for Learning: Theory and practice. Wakefield, MA: Center for Applied Special Tecnology, CAST.

Miles, M. y Huberman, A. (1994). Qualitative data analysims. Thousand Oaks, CA: Sage Publications.

Moliner García, O., Moliner Miravet, L. y Sanahuja Ribés, A. (2018). Percepciones sobre el concepto y la gestión de la diversidad en un centro en proceso de democratización escolar. Revista de Investigación Educativa, 36(2), 455-469. doi:10.6018/ rie.36.2.293191.

Nind, M. y Lewthwaite, S. (2018). Hard to teach: inclusive pedagogy in social science research methods education, In- 
ternational Journal of Inclusive Education, 22 (1), 74-88. doi: 10.1080/13603116.2017.1355413.

Petrie, K., Devcich, J. y Fitzgerald, H. (2018). Working towards inclusive physical education in a primary school: 'some days I just don't get it right', Physical Education and Sport Pedagogy, 23(4), 345-357. doi:10.1080/17408989.2018.1441391.

Rouse, M. (2009). Developing inclusive practice: A role for teachers and teacher education. Education in the North, 16, 6-13. Recuperado de https://www.abdn.ac.uk/eitn/journal/46

Sagner-Tapia, J. (2018). An analysis of alterity in teachers' inclusive pedagogical practices, International Journal of Inclusive Education, 22(4), 375-390. doi: 10.1080/13603116.2017.1370735.

Sandoval Mena, M., Simón Rueda, C. y Echeita Sarrionandia, G. (2018). A critical review of education support practices in Spain, European Journal of Special Needs Education. doi: 10.1080/08856257.2018.1533094.

Sheehy, K. y Budiyanto. (2015). The Pedagogic Beliefs of Indonesian Teachers in Inclusive Schools. International Journal of Disability, Development and Education, 62(5), 469-485. doi:10.10 80/1034912X.2015.1061109.

Souto-Manning, M. (2017). Generative text sets: Tools for negotiating critically inclusive early childhood teacher Education pedagogical practices. Journal of Early Childhood Teacher Education, 38(1), 79-101. doi: 10.1080/10901027.2016.1165760.

Spratt, J. y Florian, L. (2015). Inclusive Pedagogy: From learning to action. Supporting each individual in the context of 'everybody'. Teacher and Teaching Education, 49, 89-96. doi: 10.1016/j. tate.2015.03.006.
Stake, R. (2006). Multiple Case Study Analysis. New York: The Guilford

Susinos, T. y Parrilla, A. (2013). Investigación Inclusiva en Tiempos Difíciles. Certezas Provisionales y Debates Pendientes, REICE, 11(2), 88-98. Recuperado de https://revistas.uam.es/ index.php/reice/article/view/2898/3114

Tonnuci, F. (2017). A modo de introducción...La diversidad como valor en una escuela. Aula Abierta, 46, 9-12. doi: 10.17811/rifie.46.

Vigo, B. y Soriano, J. (2015). Family Involvement in Creative Teaching Practices for all in Small Rural Schools, Ethnography and Education, 10(3), 325-339. doi: 10.1080/17457823.2015.1050044.

Westbook, J. y Croft, A. (2015). Beginning to teach inclusively: An analysis of newly-qualified teacher pedagogy in lower primary classes in Tanzania. Teaching and Teacher Education, 51, 38-46. doi: 10.1016/j.tate.2015.05.0037.

Woodcock, S. y Hardy, I. (2017). Probing and problematizing teacher professional development for inclusion. International Journal of Educational Research, 83, 43-54. doi: 10.1016/j. ijer.2017.02.008.

Yin, H., Huang, S. y Chi Kin Lee, J. (2017). Choose your strategy wisely: Examining the relationships between emotional labor in teaching and teacher efficacy in Hong Kong primary school. Teaching and Teacher Education, 66, 127-136. doi: 10.1016/j.tate.2017.04.006. 